\title{
Contriving the Future by Tampering with the Past: A Deferral Tactic in Chinese Internet Censorship
}

\author{
Yu-Sung Su, ${ }^{1}$ Siyu Sun, ${ }^{1}$ Jiangrui Liu ${ }^{1}$ \\ ${ }^{1}$ Department of Political Science, Tsinghua Univeristy, \\ 153 Mingzhai, Tsinghua University, Haidian District, Beijing 100084, China \\ *To whom correspondence should be addressed; E-mail: suyusung@tsinghua.edu.cn
}

How do Chinese information inspectors censor the internet? In light of the assumption that inspectors must follow specific rules instead of ambiguous guidelines, such as precluding collective action, to decide what and when to delete, this study attempts to offer a dynamic understanding of censorship by exploiting well-structured Weibo data from before and after the 2018 Taiwanese election. This study finds that inspectors take advantage of time in handling online discussions with the potential for collective action. Through this deferral tactic, inspectors make online sentiments moderately flow regarding an important political event, and thereafter, past discussions on trendy topics will be mostly removed. Therefore, reality is selectively altered; the past is modified, and the future will be remembered in a "preferable" way.

\section{Introduction}

The Chinese government has established a complex system of institutions and practices of censorship and governance, which create certain myths for scholars to explore (1-6). The Chinese government exerts control over the media and other sources of information through a network 
of departments that monitor and enforce censorship policies. The whole censorship system consists of a complicated process and various executors, making the questions of how and who exactly is doing this job almost unanswerable.

Studies have found that Chinese internet censorship strategies differ across internet companies. Each company complies with the needs or goals set by the government and laws (7). We cannot know exactly when and by whom the online discussions are dealt with, nor can we fully confirm the connection between the government and the companies behind each censorship strategy, such as when the government will step in to achieve its needs and goals of censorship. As a matter of fact, many studies stealthily proclaim that censorship is executed by the government but provide no evidence. Henceforth, in this paper, we acknowledge and conceptualize an internet information inspector as any actor who may review content and make decisions on censorship. Since unpacking the black box of the whole sensitive administrative procedure is unrealistic, our focus in this paper is on the exact censoring mechanism behind inspectors with respect to the corresponding online content.

How do Chinese information inspectors censor the internet? The existing literature has unraveled the mechanisms of China's censorship, such as creating fear through the use of laws to make people self-censor themselves, building up frictions to make it costly for people to find and spread sensitive messages, and flooding people with disperse, noisy, and distracting information to divert the public opinion. The purpose of mechanisms is to primarily defuse collective action and permit proper criticism for the purpose of sustaining authoritarian resilience. However, there must be certain rules for inspectors to follow in deciding what and when to delete. The guidelines, such as precluding collective action, are rather ambiguous and are difficult for inspectors to follow. Therefore, we believe and assume that with a task that is both complex and politically risky, there must exist a naïve rule that guides basic conduct in censorship.

In light of this assumption, this study aims to offer a dynamic understanding of censor- 
ship. We utilize the data of Sina Weibo (China's largest social media platform), which are sufficiently large to provide us with statistical power to conduct ex ante observations and ex post checks on censorship processes surrounding critical political events, including changes in content networks, the distribution of relevant deleted content, and tactics regarding deleting. Methodologically, the data used include the shares, posts and comments of 203,589 Weibo accounts (total of 349,306 posts) before and after the 2018 Taiwanese election (November 24, 2018). We perform a social network analysis using links among shares, comments and original posts before and after the election to construct the whole network of these posts. URLs are extracted to verify the retention status of posts and comments.

We find that mainstream official media accounts play the prevailing role in dominating political-related discussions and communication in the course of censorship. In addition, most importantly, inspectors capitalize on selective strategies on account that posts that contain some sensitive words and topics, such as those relating to LGBT (10) rights, will be published through censorship but are restricted from spreading. Additionally, we identify a deferral tactic that allows some politically incorrect online discussions to be published in the first place and deleted afterward. In other words, the discussions are selectively pruned and altered. As a result, they will be observed and remembered in a "preferable" way. Through their use of this tactic, we propose that Chinese inspectors prevent online collective action through gradual and partial actions, rather than through immediate and thorough actions. They confine or avert deviant posts and debates in the first place so as not to fuel the related momentum. They eventually will step in and finish their censorship jobs (e.g., deleting posts) in the same way in which they would have done so in the very beginning. 


\section{Problems with Chinese Censorship}

There is no reason to judge censorship as a means of information management. Different governments have different means of conducting censorship. Each government adopts a unique censorship system according to its national conditions (11). In general, governments screen online information and delete hazardous or untrue information, including that threatening the security of the nation, the unity of ethnic groups and the stability of society, as well as that spreading rumors, pornography, and violence. In China, the transmission of politically and socially sensitive information on social media is limited due to platform-wide keyword filters and ex post content deletion. Sometimes, when an individual posts sensitive or untrue content online, the inspector will delete or restrict the post from broadcasting and remove the individual's account (12-14).

Previous empirical studies on content moderation in Chinese social media focus mainly on the censorship of messages and the fabrication of public opinion. For example, they examine how topics are associated with the intensity and velocity of censorship (15), and the fabrication of public opinion (16). Influential studies on this topic have revealed how the Chinese censorship apparatus emphasizes the silencing of expression that could potentially lead to collective action (16-18). Furthermore, using modern computer-assisted textual analytical methods and a large-scale experimental approach, internet censorship in China aims to preclude the collective action that can occur in the real world or that may be fostered or mobilized in cyberspace, while posts criticizing officials, governments or certain policies are retained for their positive value in consultation and surveilling local governance $(18,19)$. To control social media, the Chinese government is believed not only to delete online content but also to hire internet commentators (e.g., scholars, activists, journalists, and social media participants, namely, the 50 cent party) to post favorable comments about government policies as a way to sway public opinion, rather 
than to argue and even to engage in controversial issues. With such actions, these commentators redirect public attention from criticism and central issues to cheerleading and positive discussions of valence issues. Moreover, they create alternative hot topics online to distract people from their original concerns to distort the popularity of issues with high possibilities of motivating collective action $(16,20)$.

It is impossible to identify how many and which social media posts and comments are censored by governments. There are missing data that cannot be observed. To retrieve the unobservable data, previous research actively posted comments to social media sites in China and then waited to see which of these never appeared, which of these disappeared, and which of these appeared and survived (18). By varying the content of posts across topics, previous scholars concluded that any mentions of collective action in posts are selectively suppressed. Following the same belief that Chinese censorship paradoxically leaves large footprints and so reveals the behavioral patterns and intentions of the government (16-18), we move deeper to explore the process of censorship surrounding political events, when censorship happens more often. In the end, we will unscramble the motives behind such censorship and reveal the history of disappearing posts and comments.

As an observational study with sufficiently large data, we are not immune to the problem of selection bias due to the missing unobservable data caused by censorship. Nevertheless, the reality is that it is the published content and what happens to it that drive the trend of public opinion and the possibility of collective action. The ex ante filtering techniques of inspectors prevent a great deal of content from being automatically published without being reviewed (22), but people can constantly bypass these filtering techniques with various approaches (23-28) to publish new content on a particular issue that they zealously endorse. Inspectors have to address this problem to achieve their censorship goal; that is, they have to deal with published content in one way or another. 
Furthermore, if we try to look at the content targeted by censors, it is not quite realistic to simply decide what to delete. It is unmanageable for censors to differentiate what words or posts exactly are against their goals to stop criticism of the government, leaders, and policies and to put a stop to collective action. Therefore, one way to manage such an unmanageable task is to have a simple rule of basic censorship conduct with a clear goal: delete anything that either has a negative impact on society and the state or is not politically correct. Nevertheless, the problem remains that, sometimes, posts of this sort still manage to bypass censorship. In this paper, we seek to unearth how censorship is carried out under this situation. We conjecture that time is the crux that provides a buffer zone for censors in making adaptive and necessary moves and eventually accomplishing their censorship work.

\section{Data and Methods}

Internet censorship takes place with the click of a mouse. The monitoring of online content is a nearly infeasible task. To make our observational analysis tenable, in every minute between October and December 2018, we scraped the data of posts related to Taiwan and the comments and shares associated with these posts from Sina Weibo (29). Two events-the Golden Horse Film Festival and Awards (on November 17, 2018) and the 2018 Taiwan election (30) (on November 24, 2018) — are zealously discussed and create two spikes in the data (see Fig. 1).

We choose to analyze the second spike instead of the first because the election was highly political and the festival was mixed with entertaining news and gossips about movie stars. In other words, we make such a choice simply to rule out any potential confounding factors that are less relevant to our study (31).

The data in the analysis include online discussions before and after the 2018 Taiwan election, which showed an increase in online public opinion of China toward Taiwan and the crossStrait relationship. Any discussion of the cross-Strait relationship, which might negatively in- 


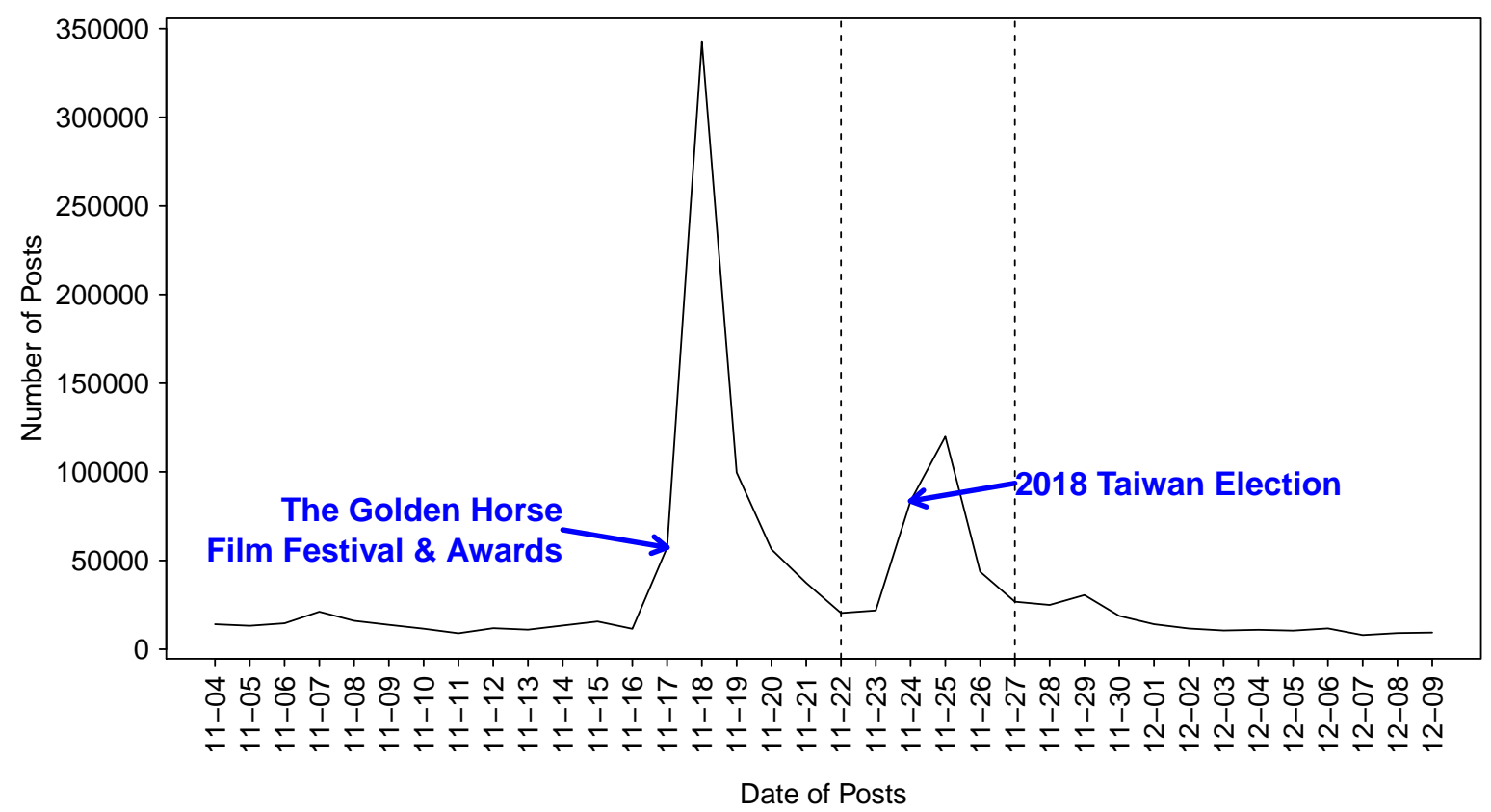

Fig. 1. The plot of the number of Weibo posts by date. Two spikes were observed near election day. We chose to ignore the first spike simply because it was less political and concerned the Golden Horse Film Festival and Awards, which is an awards festival for movies produced in Mainland China, Hong Kong, Macau, and Taiwan. The second spike concerned the election.

fluence its unification with China, often triggers the Chinese government to censor. Henceforth, if censorship did happen in the study period, then we will be able to observe how it was carried out. We define November 22-24, the first three days of the election, as Time 1, and November 25-27, the three days after the election, as Time 2 to conduct ex ante and ex post comparisons. Fig. 1 shows that these six days correspond to the ebb and flow of the online discussions. We have a total of 33,057 Weibo posts and 316,249 comments, belonging to 203,589 unique ID accounts.

We further extract data for the convenience of social network analysis. Based on the data, the "poster ID" is extracted as a unique identification code used to build nodes in the social network analysis. When an ID posts multiple messages at the same time, it is regarded as the 
interaction between a node and other information. We intercept the "post location" from the post and use only provincial information to construct Fig. 2 .

We build the network connection by using "poster IDs" as source nodes and "original poster IDs" as target nodes. Original posts with no connection are omitted. We implement the social network analysis and plot the network diagrams using Gephi 0.9.2 with the Force Atlas 2 layout algorithm.

Furthermore, we verified whether each of those posts, comments and shares still existed by revisiting their URLs in October 2019.

\section{Result and Findings}

There is a clear pattern in terms of the geographical locations of posts. Fig. 2a displays that most discussions with regard to the 2018 Taiwanese election were posted from coastal areas in China. Overseas users and users with unidentifiable locations also contributed significantly to the volume of posts during this period (approximately 30\%). Fig $2 \mathrm{~b}$ shows a similar pattern, indicating that the geographical locations of the posts before and after the election date (November 24, 2018) did not vary much. This finding may simply imply that had there existed some censorship occurring with regard to this election during these six days, then the action was uniformly carried out across China.

Fig. 3 demonstrates the whole network structure of the posts between November 22 and 27, 2018, which reveals a clear structural change in the networks. With the large amount of data in the analysis (150 thousands data points in each time period), we observe a hairball-like structure both in Figs. 3a and 3b. Nodes with 1 degree (a node links or is linked to another node) are masked from the whole network, which leaves us with $1 \%$ of the data, to better reveal the whole structure. Nevertheless, we can still identify the transformation of the structure from a polycentric one in November 22-24 to a monocentric one in November 22-27. The 


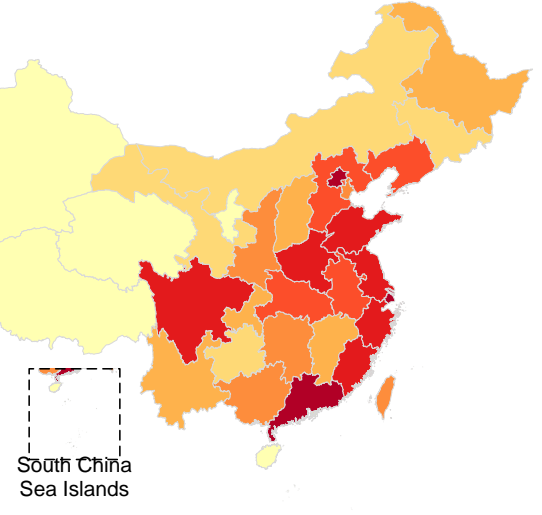

(a) November 22-24, 2018

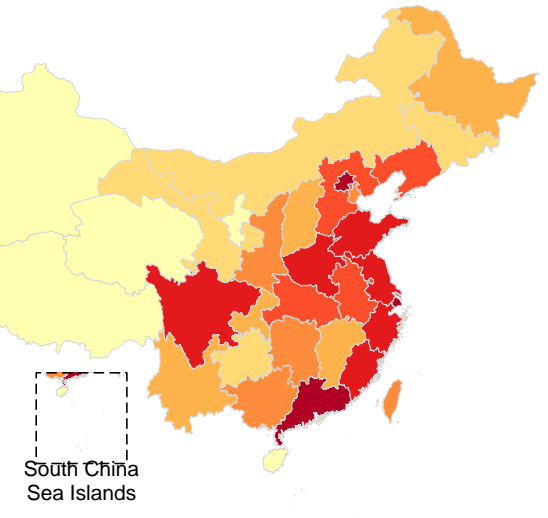

(b) November 25-27, 2018

Fig. 2. The heatmap of the geographical distribution of Weibo posts. The dark red represents the highest frequency, while the light yellow indicates the lowest frequency. There is a significant number of posts $(30 \%)$ from overseas and unknown areas, which are not shown in Fig. 2.

network in Fig. 3a is rather dispersed and has two clear clusters of nodes (colored in yellow and green), which are distant from each other (33). Conversely, the network in Fig. $3 b$ is heavily concentrated with a major cluster in the center.

This evolutionary change from Time 1 to Time 2 indicates that the discussions were pluralistic in the very beginning and became monotonic afterwards, which is a clear sign of censorship. In both Figs. $3 \mathrm{a}$ and $3 \mathrm{~b}$, the dominant nodes (i.e., the opinion leaders) are always the official media, suggesting that with regard to the posts in these two time periods, the official media dominated the online discussion on Weibo.

In both periods, the official media always dominated the discussions (see Table 1). The official media has higher degrees of involvement before and after the election, and its involvement has increased significantly after the event. Moreover, content posted by personal accounts with higher degrees of involvement was either deleted or prohibited from receiving further comments (34). In particular, LGBT issues stood out as a heat topic for discussion in these time periods. Nevertheless, such content was purged from Weibo afterward, as we revisited these 


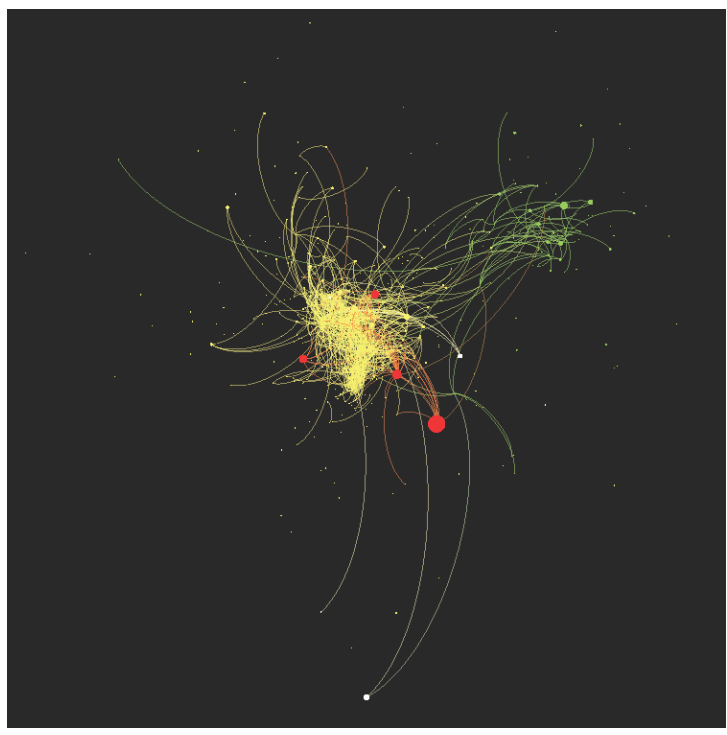

(a) November 22-24, 2018

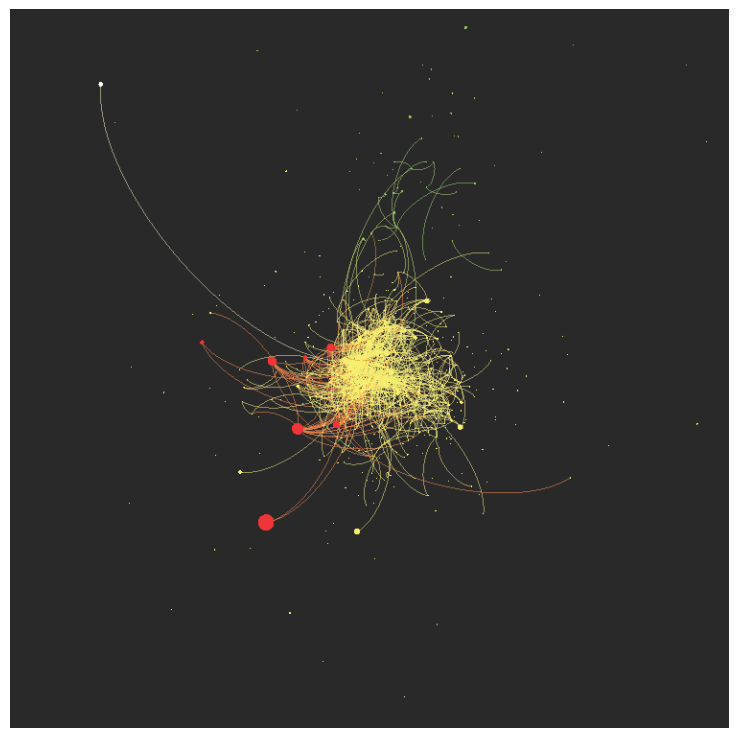

(b) November 25-27, 2018

Fig. 3. Plots of the social network analysis. The network structure in the left panel has changed from a polycentric one to a monocentric one, as shown in the right panel. The size of the node is expanded according to its degree. Two major clusters are identified based on modularity statistics (32) and are colored in yellow and green. We mark the official media in red with highest degrees from the top 20 nodes. Nodes with only 1 degree are masked from the whole network to better reveal the whole network.

posts in October 2019.

In short, we have identified a structural change through social network analysis before and after the 2018 Taiwanese election. This finding suggests a sign of censorship that trimmed the polycentric network down to a monocentric network dominated by the official media. After revisiting the posts, we have identified the further filtering of online content. One-fifth of the posts had been either removed or restricted from receiving further comments (see Table 2).

Surely, censorship existed during the study period. The reason that some sensitive content fell under the censorship radar in the first place remains an intriguing topic We will use the online discussion of LGBT issues in the following sections to elaborate on this deferral tactic in Chinese censorship. 
(a) November 22-24, 2018

\begin{tabular}{|c|c|c|c|c|c|}
\hline $\begin{array}{l}\text { Account } \\
\text { Type }\end{array}$ & $\begin{array}{c}\text { Nov. 22-24 } \\
\text { Degree }\end{array}$ & $\begin{array}{c}\text { Nov. } 25-27 \\
\text { Degree }\end{array}$ & $\Delta \%$ & $\begin{array}{l}\text { Current } \\
\text { State }\end{array}$ & Topic \\
\hline Official Media & 5296 & 7243 & $37 \%$ & Reserved & 2018 election \\
\hline Official Media & 2795 & 2704 & $4 \%$ & Reserved & 2018 election \\
\hline Official Media & 2378 & 3438 & $45 \%$ & Reserved & 2018 election \\
\hline Official Media & 2302 & 3934 & $71 \%$ & Reserved & 2018 election \\
\hline Personal & 2132 & 805 & $-62 \%$ & Deleted & LGBT issues \\
\hline Media & 1775 & 1544 & $-13 \%$ & Deleted & 2018 election \\
\hline Media & 1573 & 793 & $-50 \%$ & Reserved & LGBT issues \\
\hline Media & 1484 & 5084 & $243 \%$ & Reserved & 2018 election \\
\hline Personal & 1326 & 58 & $-96 \%$ & Comment Prohibited & LGBT election \\
\hline Personal & 1316 & 2311 & $76 \%$ & Comment Prohibited & 2018 election \\
\hline Personal & 1261 & 1939 & $54 \%$ & Reserved & 2018 election \\
\hline Personal & 1200 & 905 & $-25 \%$ & Reserved & LGBT issues \\
\hline Official Media & 1163 & 1638 & $41 \%$ & Reserved & 2018 election \\
\hline Personal & 1044 & 193 & $-82 \%$ & Deleted & LGBT issues \\
\hline Official Media & 1029 & 1574 & $53 \%$ & Reserved & 2018 election \\
\hline Media & 980 & 1069 & $9 \%$ & Reserved & 2018 election \\
\hline Personal & 974 & 315 & $-68 \%$ & Comment Prohibited & LGBT issues \\
\hline Personal & 962 & 2275 & $136 \%$ & Reserved & 2018 election \\
\hline Personal & 851 & 315 & $-63 \%$ & Comment Prohibited & LGBT issues \\
\hline Personal & 847 & 85 & $-90 \%$ & Deleted & Taiwan independence \\
\hline
\end{tabular}

(b) November 25-27, 2018

\begin{tabular}{|c|c|c|c|c|c|}
\hline $\begin{array}{l}\text { Account } \\
\text { Type }\end{array}$ & $\begin{array}{c}\text { Nov. 22-24 } \\
\text { Degree }\end{array}$ & $\begin{array}{c}\text { Nov. 25-27 } \\
\text { Degree }\end{array}$ & $\Delta \%$ & $\begin{array}{l}\text { Current } \\
\text { State }\end{array}$ & Topic \\
\hline Official Media & 5296 & 7243 & $37 \%$ & Reserved & 2018 election \\
\hline Official Media & 1484 & 5084 & $243 \%$ & Reserved & 2018 election \\
\hline Official Media & 2302 & 3934 & $71 \%$ & Reserved & 2018 election \\
\hline Official Media & 2378 & 3438 & $45 \%$ & Reserved & 2018 election \\
\hline Official Media & 2795 & 2904 & $4 \%$ & Reserved & 2018 election \\
\hline Personal & 1316 & 2311 & $76 \%$ & Reserved & 2018 election \\
\hline Personal & 962 & 2275 & $136 \%$ & Reserved & Gold Horse Award \\
\hline Personal & 0 & 2191 & $\mathrm{~N} / \mathrm{a} \%$ & Comment Prohibited & 2018 election \\
\hline Personal & 135 & 2024 & $1399 \%$ & Deleted & 2018 election \\
\hline Personal & 29 & 2002 & $6803 \%$ & Reserved & Taiwan Nuclear Energy \\
\hline Personal & 1261 & 1939 & $54 \%$ & Reserved & 2018 election \\
\hline Media & 438 & 1834 & $319 \%$ & Reserved & 2018 election \\
\hline Media & 2 & 1730 & $86400 \%$ & Reserved & Earthquake \\
\hline Official Media & 1163 & 1637 & $41 \%$ & Reserved & 2018 election \\
\hline Official Media & 1029 & 1574 & $53 \%$ & Reserved & 2018 election \\
\hline Media & 1775 & 1544 & $-13 \%$ & Reserved & 2018 election \\
\hline Media & 440 & 1471 & $234 \%$ & Deleted & Taiwan independence \\
\hline Personal & 520 & 1355 & $161 \%$ & Deleted & 2018 election \\
\hline Personal & 0 & 1307 & $\mathrm{~N} / \mathrm{a}$ & Deleted & 2018 election \\
\hline Media & 312 & 1283 & $311 \%$ & Reserved & 2018 election \\
\hline
\end{tabular}

Table 1. Lists of nodes of the top 20 degrees in November 22-24 and in November 25-27, 2018. We revisited these posts in October 2019 to obtain the current states of the posts. Posts of the official media are mostly kept intact. Others are either deleted or are prohibited from comments. 


\begin{tabular}{cccc}
\hline Period & \# of Posts & \% Deleted & \% Comment Prohibited \\
\hline $11 / 22-24$ & 125,822 & $21.8 \%$ & $0.52 \%$ \\
$11 / 25-27$ & 190,427 & $21.6 \%$ & $0.54 \%$ \\
\hline
\end{tabular}

Table 2. Percentage of posts being censored. We revisited these posts in October 2019. In both periods, one-fifth of the posts were censored. Among these, $22 \%$ were deleted and $0.5 \%$ were prohibited from receiving further comments.

\section{Discussion}

Why were LGBT issues targeted? The Chinese government always faces political and social pressure when Taiwan holds an election because the election results may lead to a major policy shift in Mainland China. For instance, Taiwan had a peaceful relationship with China between 2008 and 2016 when the pro-unification Nationalist Party (the KMT) held administrative power. The situation has deteriorated since the pro-independence Democratic Progressive Party (DPP) came to power in 2016. The major disagreement across the Strait has been the fact that the DPP does not recognize the so-called "92 consensus", which is an agreement reached between the two sides in 1992, stating that Taiwan is a part of "One China".

Internationally, China is strongly defending its "One China" policy. China's expanding influence in East Asia is also a part of this policy. Taiwan's position as the new leading force regarding LGBT rights in this region, where religion and conservative governments normally keep bans in place, reflects poorly on Mainland China. A corollary follows that since Mainland China and Taiwan share the same culture and ethnicity, if Taiwan can make progress in the legalization of same-sex marriage, then it will demonstrate that same-sex marriage can be recognized in Chinese society, conflicting with the overall policy and attitude of Mainland China concerning same-sex marriage (35).

Alongside the election, Taiwan also held a referendum on ten issues; half of these issues were related to the legalization of same-sex marriage (36). This fact was bound to attract the 
attention of Mainland Chinese homosexual individuals and LGBT groups. As minorities, these groups are always looking for a platform and window to express themselves. Moreover, the homosexual community in Taiwan has strived to legalize same-sex marriage and made many preparations before this election, including demonstrations and advocacy activities. These actions have certainly attracted great attention and may even have demonstration effects on the LGBT community in Mainland China.

Lessons from the backfire of a prompt observable mass censorship of LGBT issues. Past incidents help explain more about the intentions behind the chosen tactic covered by our analysis. In April 2018, online activism concerning the LGBT community had appeared on Weibo and successfully reversed a ban on LGBT content filtering following a public outcry (37).

On April 13, Sina Weibo announced a three-month cleanup campaign targeting content considered illegal, including pornography, violence, and topics relating to homosexuality, for the purpose of creating a harmonious online environment. This move was met with intense backlash. Thousands of Weibo users used flooding hashtags such as \# IAmGay and \# I HaveGayFriends to rally against such censorship. They posted content depicting homosexuality and expressed their support for the homosexual community. Within hours of the adverse reactions from netizens, Sina Weibo chose to ban the rallying hashtag and remove most of the comments related to the subject.

This information purge generated even wider and larger public anger. On the same day, the company's NASDAQ shares plummeted amid the controversy. On April 15, the People's Daily, China's political propaganda newspaper, appeared to criticize Sina Weibo by publishing a commentary emphasizing that it is a personal choice whether to approve of homosexuality. In addition, rationally speaking, the newspaper criticized that everyone should respect other people's sexual orientation. 
Facing furious reactions and pressure from the public, on April 16, Sina Weibo stated that this cleanup campaign of homosexual content was halted. In the same vein, the inspector carried out the censorship between November 22 and 27, 2018, with regard to LGBT rights issues with reference to the past. Sometimes, real-time censorship acts as an engagement with netizens, which may further flare up and worsen the situation. Thus, delayed treatment might be a viable alternative strategy.

On account of our findings on this deferral tactic, the inspector here is Weibo, which chose not to do the same as it had done in April to avoid deteriorating public opinion by removing a great deal of influential content and allowing time to mediate the disagreement on the homosexual topic instead of deleting everything.

\section{Concluding Remarks}

Past research has established that China utilizes censorship with the intention of distorting citizens' incentives to join in collective action against the regime, whereas certain criticisms of politics and certain discussion topics were found not to be censored. By comparing the actual records of online discussions surrounding the 2018 Taiwanese election, we further conclude that Chinese censorship uses a deferral tactic in shaping the intensity of online discussion and the history of online public opinion. The inspector, either the government or a company, selectively censors the information that citizens receive and manipulate it in a way that benefits the regime.

Admittedly, the government conducts this censorship following its self-made guidelines, and companies perform censorship according to laws and directives from the government. The inspector not only selects topics but also chooses when to intervene, as shown in our research. This deferral tactic indicates that content on some sensitive topics (e.g., LGBT issues) will be published in the first place and deleted afterward, rather than deleted immediately.

Inspectors allow topics that have a risk of spurring the flow of collective action online but 
in a restricted manner. By providing restricted freedom (38), nspectors control the intensity of online discussions in an acceptable range and allow limited space for netizens to freely express themselves (39-41). In this way, we believe that the inspector consciously or unconsciously averts simultaneous extreme posts and debates due to complaints about censorship during important political events and to prevent online collective action through gradual and partial actions.

Theoretically speaking, with this deferral tactic, the mechanisms of flooding, friction, and distraction can work even more smoothly and easily. By creating fear through the use of laws to make people self-censor themselves, building up friction to make it costly for people to find and spread sensitive messages, and flooding them with disperse, noisy, and distracting information to divert public opinion, the government can realize its censorship goals. However, when facing a large project for inspectors to work on with vast online information appearing on various Chinese platforms, in what way could the inspector possibly conduct censorship? It is believed that people's experiences of being censored undermine Chinese online censorship laws and can inspire more criticism of and discussion on sensitive issues online in China, as a form of backfire, which can result from such expression control $(42,43)$. Given that time can solve partial problems, allowing the existence of online discussions concerning sensitive topics cannot give rise to online activism without prompt massive censorship, but as a first step, inspectors censor only some leading actors by avoiding overstimulating them to bring about a negative influence on the government. By adding this tactic of time before other mechanisms, the goals of censorship collide with providing the freedom of online expression.

From the perspective of limited capability (44) to censor everything, regardless of how advanced the censor equipment held by the inspector is, it can be assumed that the government has to do the job either actively or passively, that is, to partly censor actively and to passively censor in a deferral way because the inspector, as part of an administrative machine, has to spend time 
and energy identifying what exactly triggers collective action. Our hypothesized standards of guiding censorship are simple: the inspector who scrutinizes is supposed to remove everything considered against the law or governmental directives. Nevertheless, there are still delays in terms of censorship, the logic being that Chinese internet censorship is strategic and selective.

The inspector conducts a preliminary deletion based on the impact of the content and a delayed cleanup of the entire content later on to avoid stimulating topics with real collective action potential or online collective activism potential going viral and becoming more tactful and more responsive to the censorship of public opinion. In light of this conclusion, we can obtain the practical logic of Chinese internet censorship.

Finally, we infer that the inspector always works to create a field of online public opinion that is dominated by official opinions or public opinion mainstream social norms and morals, considering that the mainstream official media dominated online discussion and communication during the 2018 Taiwanese election. Seeing the ex post disappearance of the discussions on LGBT issues in the network, when searching for keywords after such an event period, people will be scanning an altered record of what was happening during past political events. In addition, as time passes, the accumulation of online information could form a distribution in which most of the discussions on certain issues are positive, proper, and harmonious. In the end, the history of online discussions can be reconstructed rather than remaining as it is. Even-

tually, reality is selectively altered; the past is modified, and the future will be remembered in a "preferable" way. In doing so, the inspector contrives the future by tampering with the past through the use of censorship.

\section{References and Notes}

1. D. Bamman, B. O'Connor, N. Smith, Censorship and deletion practices in Chinese social media. First Monday 17 (2012). doi:10.5210/fm.v17i3.3943. 
2. G. C. Feng, S. Z. Guo, Tracing the route of China's internet censorship: An empirical study. Telematics and Informatics 30, 335-345 (2013). doi:j.tele.2012.09.002.

3. P. Lorentzen, China's strategic censorship. American Journal of Political Science 58, 402414 (2014). doi:10.1111/ajps.12065.

4. D. Wang, G. Mark, Internet censorship in China: Examining user awareness and attitudes. ACM Trans. Comput.-Hum. Interact. 22, 31:1-31:22 (Nov. 2015). doi:10.1145/2818997.

5. F. Yang, Rethinking China's internet censorship: The practice of recoding and the politics of visibility. New Media \& Society 18, 1364-1381 (2016). doi:10.1177/ 1461444814555951.

6. R. Maria, Media Politics in China: Improvising Power Under Authoritarianism, (Cambridge University Press, Cambridge, UK, 2017).

7. Censorship has been decentralized with wide variation from company to company; see $(8,9)$.

8. R. MacKinnon, China's censorship 2.0: How companies censor bloggers. First Monday 14 (2009). doi:10.5210/fm.v14i2.2378.

9. B. Miller, The limits of commercialized censorship in China (January 2019). doi:10.31235/ osf.io/wn7pr.

10. LGBT stands for lesbian, gay, bisexual and transgender.

11. J. Pan, How market dynamics of domestic and foreign social media firms shape strategies of internet censorship. Problems of Post-Communism 64, 167-188 (2017). doi:10.1080/ 10758216.2016.1181525.

12. Q. Tai, China's media censorship: A dynamic and diversified regime. Journal of East Asian Studies 14, 185-210 (2014). doi:10.1017/S1598240800008900.

13. R. Creemers, Cyber China: Upgrading propaganda, public opinion work and social management for the twenty-first century. Journal of Contemporary China 26, 85-100 (2017). doi:10.1080/10670564.2016.1206281.

14. L. Shao, The dilemma of criticism: Disentangling the determinants of media censorship in China. Journal of East Asian Studies 18, 279-297 (2018). doi:10.1017/jea.2018.19.

15. T. Zhu, D. Phipps, A. Pridgen, J. R. Crandall, D. S. Wallach, The velocity of censorship: High-fidelity detection of microblog post deletions. In Presented as part of the 22nd USENIX Security Symposium (USENIX Security 13), pp. 227-240, (USENIX, Washington, D.C., 2013). 
16. G. King, J. Pan, M. E. Roberts, How the Chinese government fabricates social media posts for strategic distraction, not engaged argument. American Political Science Review 111, 484-501 (2017). doi:10.1017/S0003055417000144.

17. G. King, J. Pan, M. E. Roberts, How censorship in China allows government criticism but silences collective expression. American Political Science Review 107, 326-343 (2013). doi:10.1017/S0003055413000014.

18. G. King, J. Pan, M. E. Roberts, Reverse-engineering censorship in China: Randomized experimentation and participant observation. Science 345, 1251722 (2014). doi:10.1126/ science. 1251722 .

19. D. D. Gueorguiev, E. J. Malesky, Consultation and selective censorship in China. The Journal of Politics 81, 1539-1545 (2019). doi:10.1086/704785.

20. R. Han, Defending the authoritarian regime online: China's "voluntary fifty-cent army". The China Quarterly 224, 1006-1025 (2015). doi:10.1017/S0305741015001216.

21. K. Fu, C. Chan, M. Chau, Assessing censorship on microblogs in China: Discriminatory keyword analysis and the real-name registration policy. IEEE Internet Computing 17, 4250 (2013). doi:10.1109/MIC.2013.28.

22. Content on social media is either routinely self-censored during the editorial process or censored and filtered according to laws; see (21)

23. Q. Yang, Y. Liu, What's on the other side of the great firewall? Chinese web users' motivations for bypassing the internet censorship. Comput. Hum. Behav. 37, 249-257 (2014). doi:10.1016/j.chb.2014.04.054.

24. H. Taneja, A. X. Wu, Does the great firewall really isolate the Chinese? integrating access blockage with cultural factors to explain web user behavior. The Information Society $\mathbf{3 0}$, 297-309 (2014). doi:10.1080/01972243.2014.944728.

25. Y. Mou, K. Wu, D. Atkin, Understanding the use of circumvention tools to bypass online censorship. New Media \& Society 18, 837-856 (2014). doi:10.1177/1461444814548994.

26. S.-y. Lee, Surviving online censorship in China: Three satirical tactics and their impact. The China Quarterly 228, 1061-1080 (2016). doi:10.1017/S0305741016001454.

27. Z.-J. Zhong, Does the great fire wall cause self-censorship? the effects of perceived internet regulation and the justification of regulation. Internet Research 27, 974-990 (2017). doi: 10.1108/IntR-07-2016-0204.

28. W. R. Hobbs, M. E. Roberts, How sudden censorship can increase access to information. American Political Science Review 112, 621-636 (2018). doi:10.1017/ S0003055418000084. 
29. This is the largest social media platform in China. The monthly active users in 2018 numbered over 445 million.

30. The purpose of the election was to elect county magistrates (city mayors), county (city) councilors, township mayors, township councilors and chiefs of village (borough) in $6 \mathrm{mu}$ nicipalities and 16 counties (cities). A multiquestion referendum was also held alongside the election. The referendum was not binding; however, it could influence the subsequent relevant policies, which could also be consequential. Elections in Taiwan usually can decisively affect Taiwan's relationship with China.

31. Nevertheless, data filtering using keywords was still required to parse out posts that were irrelevant to the keywords manually selected through rounds of tests by our research team member.

32. V. D. Blondel, J.L. Guillaume, R. Lambiotte, E. Lefebvre, Fast unfolding of communities in large networks. Journal of Statistical Mechanics: Theory and Experiment 2008, 10008 (2018). doi:10.1088/1742-5468/2008/10/P10008.

33. We use the algorithm by Blondel et al. (32) to calculate the modularity statistics to cluster nodes into several classes.

34. We revisited these posts in October 2019. The check reveals 3 conditions: currently retained, unable to leave a message, and deleted (regarded as deleted for a period of time after the event). The first two refer to the published content being merely visible to the corresponding account, thus having no chance of influencing anymore.

35. As in many other Western and non-Western societies, public sentiment regarding homosexuality in China sits within a limited space. Homosexuality has been a controversial topic across both the legal and moral domains in China. Homosexual acts were officially decriminalized in 1997. In 2001, homosexuality was removed from the official list of mental illnesses in China. However, Chinese laws have yet to properly recognize or protect samesex couples in the form of marriage. While it is not outright condemned, neither is it fully accepted as being part of the social norm.

36. Taiwanese voters faced lengthy queues as they dealt with complex ballots that featured 10 referendums, including pro- and antihomosexual marriage votes and a bid to change the name under which Taiwan competes at international sports events, which agitated China. A total of ten questions appeared on the ballot. Under Taiwanese law, a total of 280,000 signatures (1.5\% of eligible voters) were required for a question to be considered by the Central Election Commission (CEC) and presented to voters. Five of the questions reviewed and approved by the CEC concerned LGBT rights, LGBT sex education and same-sex marriage. 
37. S. Liao, “\#IAmGAY\# what about you?": Storytelling, discursive politics, and the affective dimension of social media activism against censorship in China. International Journal of Communication 13 (2019).

38. J. Lu, Y. Zhao, Implicit and explicit control: Modeling the effect of internet censorship on political protest in China. International Journal of Communication 12 (2018).

39. B. Qin, D. Strömberg, Y. Wu, Why does China allow freer social media? protests versus surveillance and propaganda, Journal of Economic Perspectives 31, 117-40 (February 2017). doi:10.1257/jep.31.1.117.

40. J. Chen, Y. Xu, Information manipulation and reform in authoritarian regimes. Political Science Research and Methods 5, 163-178 (2017). doi:10.1017/psrm.2015.21.

41. H. Huang, S. Boranbay-Akan, L. Huang, Media, protest diffusion, and authoritarian resilience. Political Science Research and Methods 7, 23-42 (2019). doi:10.1017/psrm. 2016.25 .

42. M. E. Roberts, Censored: Distraction and Diversion Inside China's Great Firewall, (Princeton University Press, Princeton, NJ, 2018).

43. N. F. Johnson, R. Leahy, N. J. Restrepo, N. Velasquez, M. Zheng, P. Manrique, P. Devkota, S. Wuchty, Hidden resilience and adaptive dynamics of the global online hate ecology. Nature 573, 261-265 (2019). doi:10.1038/s41586-019-1494-7.

44. Even with real name registration and increasing numbers of arrests, enforcement against all users who violate China's broad internet laws is difficult for the government to carry out; see (42). 